\title{
Elastic and inelastic scattering as a means of identifying shell closures
}

\author{
S. Karataglidis ${ }^{1, a}$, K. Amos ${ }^{2}$, and B.A. Brown ${ }^{3}$ \\ ${ }^{1}$ Department of Physics and Electronics, Rhodes University, 6140 Grahamstown, South Africa \\ 2 School of Physics, University of Melbourne, 3010 Victoria, Australia \\ 3 NSCL and Department of Physics and Astronomy, Michigan State University, S. Shaw Lane, East Lansing 48824-1321, USA
}

\begin{abstract}
It has been suggested that the magic numbers in nuclei are modified as one approaches the drip lines from the valley of stability. It is proposed that elastic and inelastic proton scattering along the isotope chain to the drip line may be a good means to observe this. Using the even-mass Carbon isotopes as an example, we find that the elastic cross sections define the mass-changing character of the nuclei. Those of the inelastic scattering to the $2_{1}^{+}$states reflect strongly the neutron shell closure at ${ }^{14} \mathrm{C}$. Implications for future experiments are discussed.
\end{abstract}

\section{Introduction}

There has been much speculation concerning the possible melting or changing of the shell structure of nuclei as one moves away from the valley of stability. Indications are that some of those changes may go beyond a simple Nilsson scheme whereby they are indicative of modifications in the single particle energies. That there are indications beyond Nilsson-type deformations in the single particle energies comes from a measurement of $(\alpha, \mathrm{t})$ reactions on the eveneven stable $\mathrm{Sn}$ isotopes [1], the results suggesting a possible decrease in the spin-orbit potential with increasing neutron excess. However, that study was limited to heavy nuclei and no such change in the spin-orbit potential has been found in the light nuclei. Indications of possible changes in the magic numbers have also been postulated. There are indications of such around ${ }^{32} \mathrm{Mg}$ giving rise to the observed "island of inversion" [2]. Inclusion of three-body forces may also play a role $[3,4]$. There is also evidence that changes to the single particle energies as one approaches the drip lines, due to changes in the monopole term of the Hamiltonian, may lead to new magic numbers [5].

Were there to be such an extreme change in the shell structure away from stability, one should seek a means by which to identify it. One method is to consider the energy of the first $2^{+}$state systematically about a suspected closed shell [6]. At a closed shell, the energy of the $2_{1}^{+}$state in that nucleus is much larger than those in neighboring nuclei. Such was used to identify a new $N=32$ subshell closure in ${ }^{56} \mathrm{Cr}$ [6].

The Carbon isotopes provide an environment whereby one may observe a trend from the proton $\left({ }^{10} \mathrm{C}\right)$ to the neutron $\left({ }^{18} \mathrm{C}\right)$ drip lines. The $B(E 2)$ value is an observable which may show such a trend and one expects there to be a significant change at the shell closure given the change in energy of the $2^{+}$state. It would also be useful to consider observables at finite momentum transfer, as such may vary greatly and lead to significant effects. Herein, we look to scattering to investigate a means by which shell closures and crossing may be identified, and in particular consider proton scattering at intermediate energies. At these energies, the nucleon-nucleon

${ }^{a}$ Presenting author, e-mail: S.Karataglidis@ru.ac.za
$(N N)$ potential is dominated by the $V_{p n}$ component interaction [7] and so proton scattering primarily, though not exclusively, probes the neutron density and vice-versa. This then also allows for investigation of shell closures of either the proton or neutron density independently. The neutron shell closure in the $\mathrm{C}$ isotopes occurs at ${ }^{14} \mathrm{C}$, with a doubly closed $0 p$ shell. There is closure of the $0 p_{\frac{3}{2}}$ shell in ${ }^{12} \mathrm{C}$, but that is not a purely closed subshell as there are significant $2 \mathrm{p}-2 \mathrm{~h}$ terms in the wave function. Also, we consider inelastic scattering leading to the first $2^{+}$state in each isotope, as there may be a significant change at the shell closure consistent with the change in energy. Any change would be expected to occur in both elastic and inelastic scattering around ${ }^{14} \mathrm{C}$.

\section{Intermediate energy scattering from Carbon}

To predict the differential cross sections for both elastic and inelastic scattering from the $\mathrm{C}$ isotopes we utilise the microscopic $g$-folding model of the Melbourne group [7]. That model begins with the $N N g$ matrices for the interaction of a nucleon with infinite nuclear matter. (The $N N$ interaction used herein is the BonnB interaction [8].) Those $g$ matrices are solutions of the Brueckner-Bethe-Goldstone equations in infinite nuclear matter, viz.

$$
\begin{aligned}
& g\left(\mathbf{q}^{\prime}, \mathbf{q} ; K\right)=V\left(\mathbf{q}^{\prime}, \mathbf{q}\right) \\
& +\int V\left(\mathbf{q}^{\prime}, \mathbf{k}^{\prime}\right) \frac{Q\left(\mathbf{k}^{\prime}, \mathbf{K} ; k_{f}\right)}{\left[E(\mathbf{k}, \mathbf{K})-E\left(\mathbf{k}^{\prime}, \mathbf{K}\right)\right]} g\left(\mathbf{k}^{\prime}, \mathbf{q} ; \mathbf{K}\right) d \mathbf{k}^{\prime},
\end{aligned}
$$

where $Q$ is a Pauli-blocking operator and the effects of the nuclear medium are introduced via the auxiliary potentials in the energy denominator [7]. $K$ and $k_{f}$ are the center-of-mass and Fermi momenta, respectively. The $g$ matrices are then mapped via a double Bessel transform to equivalent forms in coordinate space. A local density approximation maps those to the effective interactions in finite nuclei. Folding those effective interactions with the density matrices of the target then yields the nonlocal, density-dependent, nucleon-nucleus $(N A)$ optical potential, from which the elastic scattering 
observables are obtained. Full details are found in the review article [7] to which the reader is referred.

Inelastic scattering is calculated within the DistortedWave-Approximation (DWA) using the effective coordinate space $g$ matrices as the transition operator. The transition amplitude is given by

$$
T_{J_{f} J_{i}}^{M_{f} M_{i} v^{\prime} v}(\theta)=\left\langle\chi_{v^{\prime}}^{(-)}\right|\left\langle\Psi _ { J _ { f } M _ { f } } \left| A g_{\mathrm{eff}}(0,1) \mathcal{A}_{01}\left\{\left|\chi_{v}^{(+)}\right\rangle\left|\Psi_{J_{i} M_{i}}\right\rangle\right\}\right.\right.
$$

where $\chi^{( \pm)}$denoted the distorted wave functions for an incoming/outgoing nucleon, respectively, and 0,1 denote the coordinates of the projectile and bound state nucleon, respectively. By using a co-factor expansion of the target wave function one obtains

$$
\begin{aligned}
& T_{J_{f} J_{i}}^{M_{f} M_{i} v^{\prime} v}(\theta) \\
& \quad=\sum_{\alpha_{1} \alpha_{2} m_{1} m_{2}} \sum_{J M} \frac{(-1)^{j_{1}-m_{1}}}{\sqrt{2 J_{f}+1}}\left\langle j_{2} m_{2} j_{1}-m_{1} \mid J_{f} M_{f}\right\rangle \\
& \quad \times\left\langle J_{i} M_{i} J M \mid J_{f} M_{f}\right\rangle\left\langle J_{f}\left\|\left[a_{\alpha_{2}}^{\dagger} \times \tilde{a}_{\alpha_{1}}\right]^{J}\right\| J_{i}\right\rangle \\
& \quad \times\left\langle\chi_{v^{\prime}}^{(-)}(0)\right|\left\langle\varphi _ { \alpha _ { 2 } } ( 1 ) \left| A g_{\mathrm{eff}}(0,1) \mathcal{A}_{01}\left\{\left|\chi_{v}^{(+)}(0)\right\rangle\left|\varphi_{\alpha_{1}}(1)\right\rangle\right\}\right.\right.
\end{aligned}
$$

for an angular momentum transfer $J$, and $\alpha$ denotes the set of single-particle quantum numbers $\left\{n, l, j, m_{\tau}\right\}$, where $\tau$ is the nucleon isospin.

\section{Structures of Carbon}

For this study we have used two different models of structure. The first is the Skyrme-Hartree-Fock (SHF) model of Brown [9]. That model uses the SKX interaction to obtain the ground state densities. As the SHF model cannot provide information on the spectrum of the target, we have also used a $(0+2) \hbar \omega$ shell model. That model allows for the determination of the transition densities necessary for the calculation of inelastic scattering. Note that for ${ }^{10,12,14} \mathrm{C}$ this model is complete. The model reduces to $0 \hbar \omega$ for ${ }^{16,18} \mathrm{C}$. For the calculations presented herein, we have used the OXBASH Shell Model program [10] with the WBP interaction of Warburton and Brown [11] and with suitable corrections for the center-of-mass. From that model we have found that the ground state wave functions for ${ }^{10} \mathrm{C},{ }^{12} \mathrm{C}$ and ${ }^{14} \mathrm{C}$ are

$$
\begin{aligned}
& \left|{ }^{10} \mathrm{C}\right\rangle=92.4 \%|0 \hbar \omega\rangle+7.6 \%|2 \hbar \omega\rangle \\
& \left|{ }^{12} \mathrm{C}\right\rangle=87.0 \%|0 \hbar \omega\rangle+13.0 \%|2 \hbar \omega\rangle \\
& \left|{ }^{14} \mathrm{C}\right\rangle=84.9 \%|0 \hbar \omega\rangle+15.1 \%|2 \hbar \omega\rangle .
\end{aligned}
$$

In that same model, the states in ${ }^{16} \mathrm{C}$ and ${ }^{18} \mathrm{C}$ are purely $0 \hbar \omega$ in character.

\section{Results}

The differential cross sections for the elastic scattering of $100 \mathrm{MeV}$ protons from the even-mass Carbon isotopes using the shell occupancies and single particle wave functions

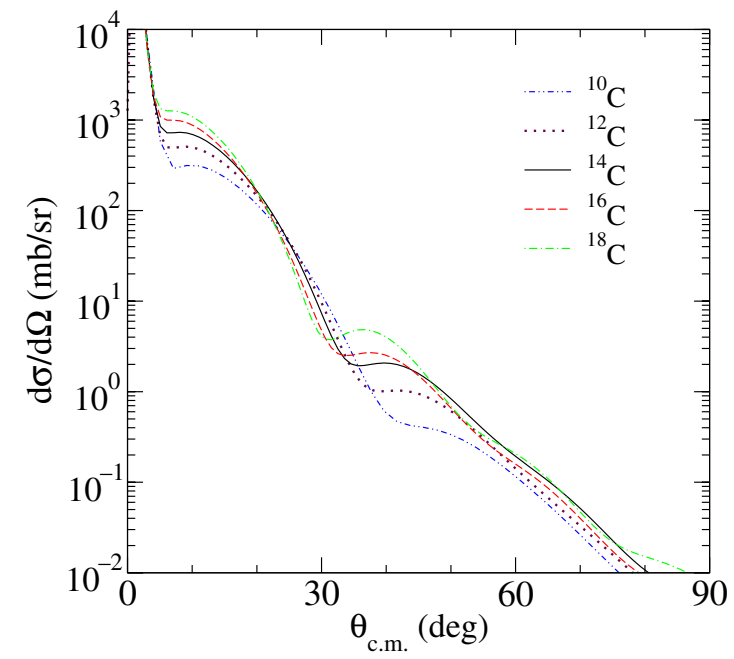

Fig. 1. (Color online.) The differential cross sections for the elastic scattering of $100 \mathrm{MeV}$ protons from the even-mass isotopes of Carbon with structure obtained from the Skyrme-Hartree-Fock model.

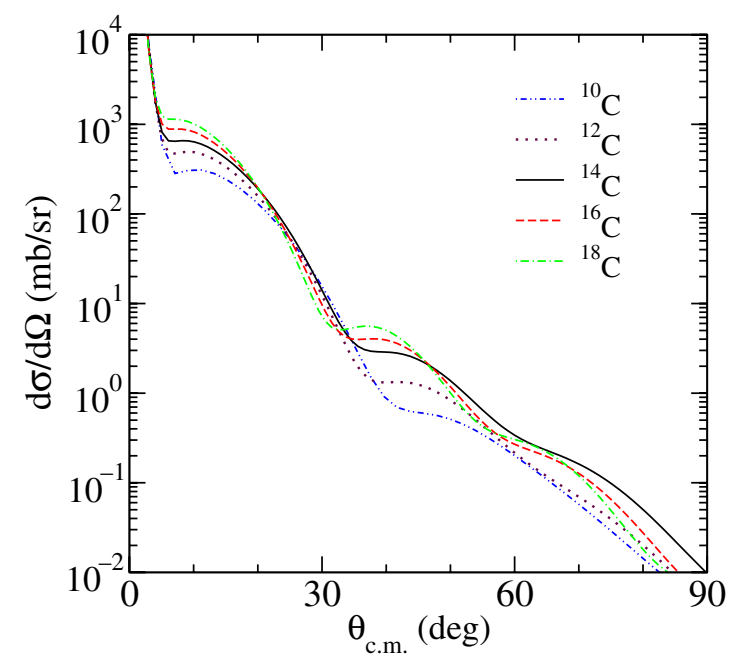

Fig. 2. (Color online.) As for figure 1, but using structures obtained from the shell model.

obtained by using the SHF model are displayed in figure 1 . There is a steady increase in the cross section with angle indicating an increase in the rms radius with mass. Beyond the first diffraction minimum the cross sections from ${ }^{10} \mathrm{C}$ and ${ }^{12} \mathrm{C}$ are significantly reduced compared to those of the other isotopes. That indicates the lack of neutron $0 p$-shell strength in the density, as ${ }^{14,16,18} \mathrm{C}$ all have a closed $0 p$ neutron shell. The differential cross sections obtained using shell model wave functions, for which an oscillator parameter of $1.65 \mathrm{fm}$ was used to specify the single-particle wave functions, are displayed in figure 2 . The same trends are observed in the cross sections obtained using the shell model as were found with the SHF model results. Note that overall, the results obtained using the shell model wave functions are slightly larger than those obtained using the SHF wave functions. This is especially so beyond the first minimum, indicating that the shell model may predict more neutron strength within 
Table 1. Energies, in $\mathrm{MeV}$, of the $2_{1}^{+}$state in the Carbon isotopes. Experimental values are from ref. [12].

\begin{tabular}{ccc}
\hline Isotope & Theory & Experiment \\
\hline${ }^{10} \mathrm{C}$ & 4.272 & 3.354 \\
${ }^{12} \mathrm{C}$ & 4.421 & 4.439 \\
${ }^{14} \mathrm{C}$ & 6.920 & 7.012 \\
${ }^{16} \mathrm{C}$ & 2.385 & 1.77 \\
${ }^{18} \mathrm{C}$ & 2.114 & $1.62^{a}$ \\
\hline
\end{tabular}

${ }^{a}$ This is a tentative assignment only [12].

the interior while the SHF model places more strength in the surface.

We now consider inelastic scattering to the first $2^{+}$state in each nucleus. Table 1 contains the shell model predictions for each state as compared to experiment [12]. The agreement between the results of the shell model calculations with data is quite good, though the $2^{+}$assignment for the $1.62 \mathrm{MeV}$ state in ${ }^{18} \mathrm{C}$ is only tentative [12]. Note that the energy of the $2^{+}$ state in ${ }^{14} \mathrm{C}$ is much larger than the other isotopes.

Using the shell model structures to define the transition OBDME, the differential cross sections for the inelastic scattering of $100 \mathrm{MeV}$ protons to the $2_{1}^{+}$state in each nucleus are displayed in figure 3 . Here, the differential cross sections from



Fig. 3. (Color online.) The differential cross sections for the inelastic scattering of $100 \mathrm{MeV}$ protons to the $2_{1}^{+}$states in ${ }^{10,12,14,16,18} \mathrm{C}$, as obtained from the shell model calculations described in the text.

${ }^{10,12} \mathrm{C}$ and ${ }^{16,18} \mathrm{C}$ while comparable in magnitude are most distinctly different in shape. That indicates the change in the density with the introduction of the $s d$-shell neutrons in ${ }^{16,18} \mathrm{C}$. Most striking is the reduction in strength at forward angles of the differential cross section from scattering to the $2^{+}$state in ${ }^{14} \mathrm{C}$. This reduction is an order of magnitude at $0^{\circ}$ decreasing to a factor of 6 at $20^{\circ}$. There is very little neutron strength in the transition density in ${ }^{14} \mathrm{C}$ as the neutron shell is closed. That translates into a reduction in the inelastic cross section compared to those from excitations of the $2^{+}$states in the other isotopes.

\section{Conclusions}

We have shown that the cross sections from inelastic scattering may be used to identify shell closure as, for the nucleus in which that occurs, the transition density can be markedly reduced from similar transition cross sections in neighboring isotopes. Beyond the shell closure, the change in the density effected by the introduction of higher-order shells is also significant. These two points together are indicative of the major shell closure. While the transition may be investigated with zero-momentum transfer observables, such as $B(E l)$ values, one may only ascertain the change in the density with data from scattering experiments that probe the density at finite momentum transfer values. It must be remembered, however, that any experimental investigation of the inelastic scattering must be accompanied by the taking of elastic scattering data in order to interpret correctly the underlying optical potentials involved [13] and so generate the appropriate distorted wave functions to be used in the analyses of inelastic scattering data.

We stress that these results are still preliminary. Further checks need to be done for consistency in the shell model for all the isotopes presented. This is especially true for ${ }^{16} \mathrm{C}$, where one must also consider the anomalous $B(E 2)$ value [14].

\section{References}

1. J.P. Schiffer et al., Phys. Rev. Lett. 92, 162501 (2004).

2. E.K. Warburton, J.A. Becker, B.A. Brown, Phys. Rev. C 41, 1147 (1990).

3. S.C. Pieper, V.R. Pandharipande, R.B. Wiringa, J. Carlson, Phys. Rev. C 64, 014001 (2001).

4. S.C. Pieper, K. Varga, R.B. Wiringa, Phys. Rev. C 66, 044310 (2002).

5. T. Otsuka, R. Fujimoto, Y. Utsuno, B.A. Brown, M. Honma, T. Mizusaki, Phys. Rev. Lett. 87, 082502 (2001).

6. J.I. Prisciandaro et al., Phys. Lett. B 510, 17 (2001).

7. K. Amos, P.J. Dortmans, H.V. von Geramb, S. Karataglidis, J. Raynal, Adv. Nucl. Phys. 25, 275 (2000).

8. R. Machleidt, K. Holinde, C. Elster, Phys. Rep. 149, 1 (1987).

9. B.A. Brown, Phys. Rev. C 58, 220 (1998).

10. OXBASH-MSU (the Oxford-Buenos-Aries-Michigan State University shell model code). A. Etchegoyen, W.D.M. Rae, N.S. Godwin (MSU version by B.A. Brown, 1986); B.A. Brown, A. Etchegoyen, W.D.M. Rae, MSUCL Report Number 524 (1986).

11. E.K. Warburton, B.A. Brown, Phys. Rev. C 46, 923 (1992).

12. TUNL Nuclear Data Evaluation, http://www.tunl.duke.edu/nucldata/index.shtml

13. K. Amos, W.A. Richter, S. Karataglidis, B.A. Brown, Phys. Rev. Lett. 96, 032503 (2006).

14. I. Imai et al., Phys. Rev. Lett. 92, 062501 (2004). 